\title{
Search for EAS candidates with the TUS /Lomonosov orbital experiment: results of preliminary data reconstruction and analysis
}

Leonid Tkachev ${ }^{1}$ on behalf the Lomonosov-UHECR/TLE collaboration

1. Joint Institute for Nuclear Research, Joliot-Curie, 6, Dubna, Moscow region, 141980 Russia,

2. Dubna State University, University str., 19, Bld.1 Moscow region, 141980 Russia

E-mail: tkatchevejinr.ru

\begin{abstract}
The "Lomonosov" satellite, with the TUS instrument on board, was launched on April 28, 2016 on solar-synchronized orbit with altitude about $500 \mathrm{~km}$. The main goal of TUS space experiment is to search Ultra High Energy Cosmic Rays (UHECR) at E > 70 EeV by measuring the fluorescence and Cherenkov radiation of extensive air showers (EASs) in the Earth's atmosphere. The TUS instrument's design and principles of operation are briefly presented. A multi-level algorithm for the search of EAS-like events was developed and applied to the TUS data set analysis. The preliminary results of the off-line reconstruction program for data analysis, search and study of candidates for the UHECR event are described.
\end{abstract}

\section{${ }^{1}$ Leonid Tkachev}

(c) Copyright owned by the author(s) under the terms of the Creative Commons 


\section{Introduction}

The measurements of Cosmic Ray (CR) spectrum, composition and anisotropy in the wide energy interval are an important part of the particle physics study. Ultra High Energy Cosmic Rays (UHECR) at E $\sim 10^{20} \mathrm{eV}$ were discovered more than 50 years ago [1], but the results in the UHECR physics do not give clear answers to the most important questions. The UHECR origin and nature, the mass composition, anisotropy and possible UHECR sources are still unclear. To a large extent, the problem is due to the very low UHECR flux - the current Auger [2] and TA [3] experiments have 200 UHECR events at E $>5 \cdot 10^{19} \mathrm{eV}$ and a few events at $\sim 10^{20} \mathrm{eV}$.

The TUS project's goal is the experimental study of UHECR. The fluorescent and Cherenkov radiation of the Extensive Air Showers (EAS) generated by UHECR particles should be detected in the Earth's atmosphere on the night side of the orbit at altitudes $400-500 \mathrm{~km}$. It will be possible to evaluate the EAS energy and arrival directions at energies $\mathrm{E}>7 \cdot 10^{19} \mathrm{eV}$ that is beyond the GZK limit [4, 5]. The SINP MSU, JINR and Space Regatta Consortium together with several Korean and Mexican Universities have collaborated in the TUS detector preparation.

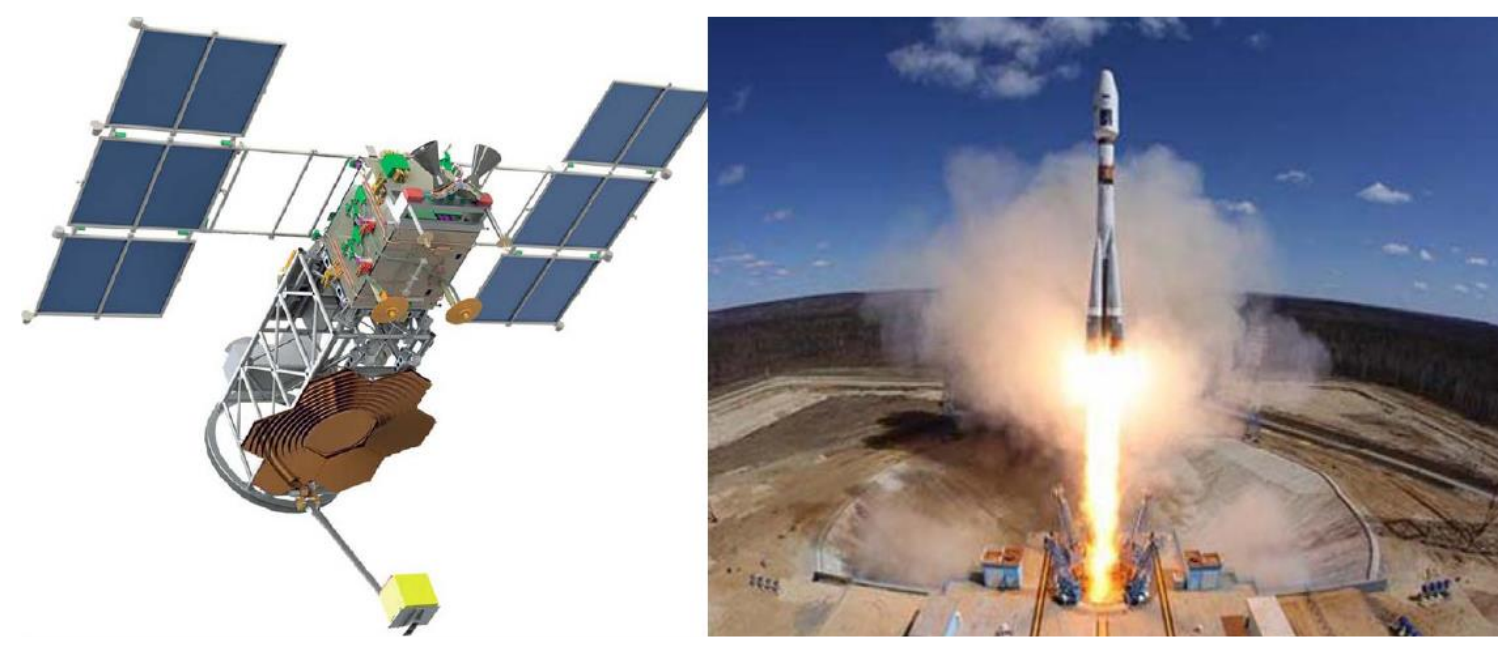

Figure 1. Left: scheme of the TUS detector on-board the "Lomonosov" satellite. Right: launch of the satellite on April 28, 2016.

An important advantage of space detector is the possibility of taking data from different arrival directions of the sky with the same apparatus and with the same systematic uncertainties. EAS photons move to detector through the atmosphere that is more transparent in the way to space-based detector in comparison with the EAS photons going down to the ground-based detectors. But, atmospheric conditions are very unstable during the flight over the different geographical locations. This leads to a change of background atmospheric radiation and an automatic system of HV regulation reacts. With such data the existent difference between the results of the Auger detector in the south hemisphere and the TA detector in the north hemisphere may be understood [6,7]. However, the EAS signal in the space orbit is $\sim 100$ times weaker in comparison with the ground-based detectors, which leads to a difficult problem due to background albedo radiation of the Earth's atmosphere. The permanent monitoring and calibration of the TUS apparatus with different ground-based calibration sources is being prepared [8]. The software package has been developed to simulate performance of the TUS detector - the TUSSIM program [9] for the Fresnel mirror optical parameters, the light guide of the photo detector, the front end and trigger electronics. 
The TUS detector was launched to the orbit with altitude $500 \mathrm{~km}$ on April 28, 2016. Being the first orbital detector of EAS study in the atmosphere it is a pathfinder for the next, larger UHECR fluorescence detectors KLYPVE [10] and JEM-EUSO [11].

\section{The TUS detector, trigger and DAQ.}

There are two main parts of the detector: a modular Fresnel mirror and a photo receiver matrix with the corresponding DAQ electronics [12] (field of view (FoV) $\pm 4.5^{\circ}$, number of pixels 16x16 and light guides). One pixel is a Hamamatsu R1463 PMT with a 13-mm-diameter tube and UV glass window. A PMT quantum efficiency is $\sim 20 \%$ for the wavelength of $350 \mathrm{~nm}$. The multi-alkali cathode was chosen for operation in linear regime in a wider range of temperatures. The Fresnel mirror has an area of $2.0 \mathrm{~m}^{2}$ and a focal distance of $1.5 \mathrm{~m}$. The DAQ electronics forms 256 channels with a time step $\Delta \mathrm{t}=0.8 \mu \mathrm{s}$. One pixel $\mathrm{FoV}$ is $\approx 10^{-4} \mathrm{sr}$, which corresponds to a spatial spot of $5 \times 5 \mathrm{~km}^{2}$ on the Earth surface for the $500 \mathrm{~km}$ orbit height and $80 \times 80 \mathrm{~km}^{2}$ for the whole detector.

The photodetector consists of 16 modules, each of 16 pixels, and the front end photodetector module (PDM) board. Each PDM has its own digital data processing system for the first-level trigger, based on XILINX FPGA, and a high voltage power supply controlled by the FPGA to adjust the PMT gain to the background intensity. The PDM board calculates the Moving Sum (MS) of the digitized PMT signal $A_{i j}(t)$ during the 16 time steps in each pixel

$$
M S_{i j}(t)=\sum_{k=0}^{15} A_{i j}(t-(15-k) \Delta t)
$$

where $\mathrm{i}, \mathrm{j}$ are the module and PMT numbers respectively.

A crucial part of the TUS detector is a trigger system. The PDM electronics produces a trigger signal of the level 1 in the module if $M S_{\mathrm{ij}}(\mathrm{t})$ value exceeds the threshold level. The threshold level is calculated as $\left\langle M S_{\mathrm{ij}}\right\rangle+d Q$, where $\left\langle M S_{\mathrm{ij}}\right\rangle$ is the mean value of $M S$ obtained during previous $100 \mathrm{~ms}$ for each channel, and $d Q$ is a unique constant for all channels.

Every 16 time steps the PDM boards transmit trigger word to the central processor board (CPB) that forms a map of $16 \times 16$ pixels where the $M S$ values were above the threshold. The level 2 trigger is generated if the hit pixels are localized in the same or neighboring bins in the $L$ (adjacency length) consecutive maps. For generation of the level 2 trigger $L=3-6$ consecutive maps are analyzed. $d Q$ and $L$ values can be set from the mission center and selected according to the atmospheric background radiation level and the trigger rate that is limited by the channel transfer capacity from the TUS CPB to the on-board computer. More detailed information about the TUS trigger work will be described below in the off-line analysis where the similar algorithms are used in the EAS events' selection of raw data. When the level 2 trigger condition is fulfilled, the raw data array of 256 PMT currents for 256 time steps is transmitted to the on-board computer from the motherboard memory. The EAS event size is 128 $\mathrm{kB}$ and the trigger rate is 1 per minute due to the limited speed of the raw data transmission. The design of the TUS detector and its performance is presented in more details in [13].

Complex mechanical and electrical tests were fulfilled with the TUS apparatus during 2014-2015 that were completed by launching on April 28, 2016 into orbit from the new Vostochny Cosmodrome as a part of the "Lomonosov" satellite as shown in the right side of Fig.1. The "Lomonosov" satellite has a solar-synchronized orbit with an inclination of $\sim 97^{\circ}$, a period of circulation $\sim 94 \mathrm{~min}$, and heights $470-500 \mathrm{~km}$.

\section{First year of TUS flight. Background investigation.}

The first months of the TUS operation in orbit were devoted to the apparatus functionality measurements. After flight tests and a tuning of TUS hardware and software on the satellite, the regular data are received since the end of August 2016. During regular operation, 
the detector measures the UV background level and adjusts the HV and sensitivity of the PMTs to avoid its saturation under the changed intensity of background radiation. The HV correction occurs once every $100 \mathrm{~ms}$ to ensure a constant anode current during EAS waveform measurements. Unfortunately, during the first days of operation several PMTs were broken due to HV tuning system failure. For the same reason, the properties of the rest of PMTs are changed and that demands a special PMT behavior investigation based on the TUS data analysis itself. Ground-based calibration sources for calibration of the TUS detector in flight have been developed and their manufacture is in progress [14]. Areas of the PMT matrix without sensitivity reduce the capability of the EAS study. Besides, some number of the noise PMTs produced continuously fake triggers that occupy the DAQ system.

A big surprise was a lot of triggers due to CR particle tracks in the UV filter in front of PMTs those are visible via the fluorescent and Cherenkov light irradiation due to interaction of the CR particle with the UV filter matter. Due to rather slow decreasing PMT current, such type events generate a number of triggers that blocked TUS data taking due to occupation of the data transfer channel between the TUS and satellite on-board computer. More information about the different types of the background events may be found in [15]. Such events are about $10 \%$ of the total number of events taken at moonless nights.

Because of solar-synchronized orbit one week before and after the moonless time there is no Moon in the sky during the night part of the TUS orbit. It means that the TUS gets $\sim 7000$ triggers/month. After removing the non-moonless files, the remaining data have been analyzed through the Event Display program.

\section{EAS event reconstruction program development.}

A number of software dedicated to the TUS data simulation, visualization and analyses were developed in JINR for EAS search. After rejection of obvious background events using the Event Display program the remaining ones are analyzed by the TUSReadData event reconstruction program in semi-automatic regime. First of all the 16 frame's event-map plot is created. Eight of such frames with hit pixels of selected EAS candidate event are presented in Fig.2. Each frame is $16 \times 16$ PMT matrix image with the hit pixels. For this, the time evolution of the PMT amplitude in the each pixel is analyzed during the 256 time steps. PMT amplitudes $A(t)$ are normalized to the mean values during the event duration $A(t) /$ Mean and the $M S$ sums are calculated. In the time dependence analysis the hit pixels are selected in the case the $M S$ sum value is above the trigger-1 value which is free parameter of the program. In the next analysis step, the TUSReadData program creates a number of "tracks" which combine hit pixels at the neighboring frames and at neighboring locations of the hit pixels in frames. At this stage, it is important to visually check the plot and evaluation of the program analysis results, including the track locations between the dead PMTs, and selection of the value of the threshold.

After the visual check, the TUSReadData program analyzes the same event again with the selected track and eliminates the others. The time dependence amplitudes $A(t)$ of the selected pixels are fitted by function $F(t)=p_{0}+\operatorname{Gauss}\left(t, A_{\max }, t_{\max }, \sigma\right)$, here $A_{\max }, t_{\max }, \sigma$ are the parameters of the gaussian approximation (maximum value, time of the maximum and its width). It is natural to consider the $p_{0}$ parameter as a background value and in the following analysis the selected pixel amplitudes are renormalized according to $A(t) /$ Mean $\rightarrow\left(A(t)-p_{0}\right) / p_{0}$. 

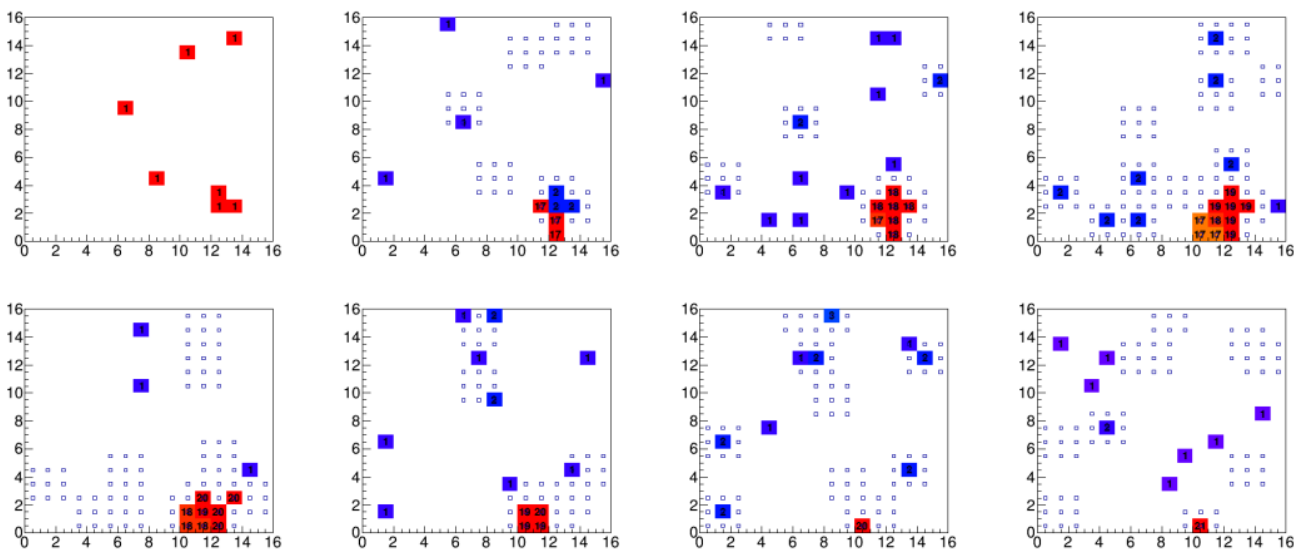

Figure 2. The 8 frames of the event-map plots of the sequential frames of $16 \times 0.8 \mu$ s steps. Small points indicates an expected location pixels with respect to previous hit ones to be included in the same track. Figures in the pixel indicate the track numbers.

To check a correctness of the program, the reconstruction analysis was fulfilled for Monte-Carlo simulated events that is generated with ESAF package [17] and the TUSSIM program [9]. An example of such event is presented in the left side of Fig.3 (energy $E=10^{20.4}$ $\mathrm{eV}$, zenith angle $\theta=66^{\circ}$ ). It shows an image (track) of the simulated EAS on the PMT matrix.
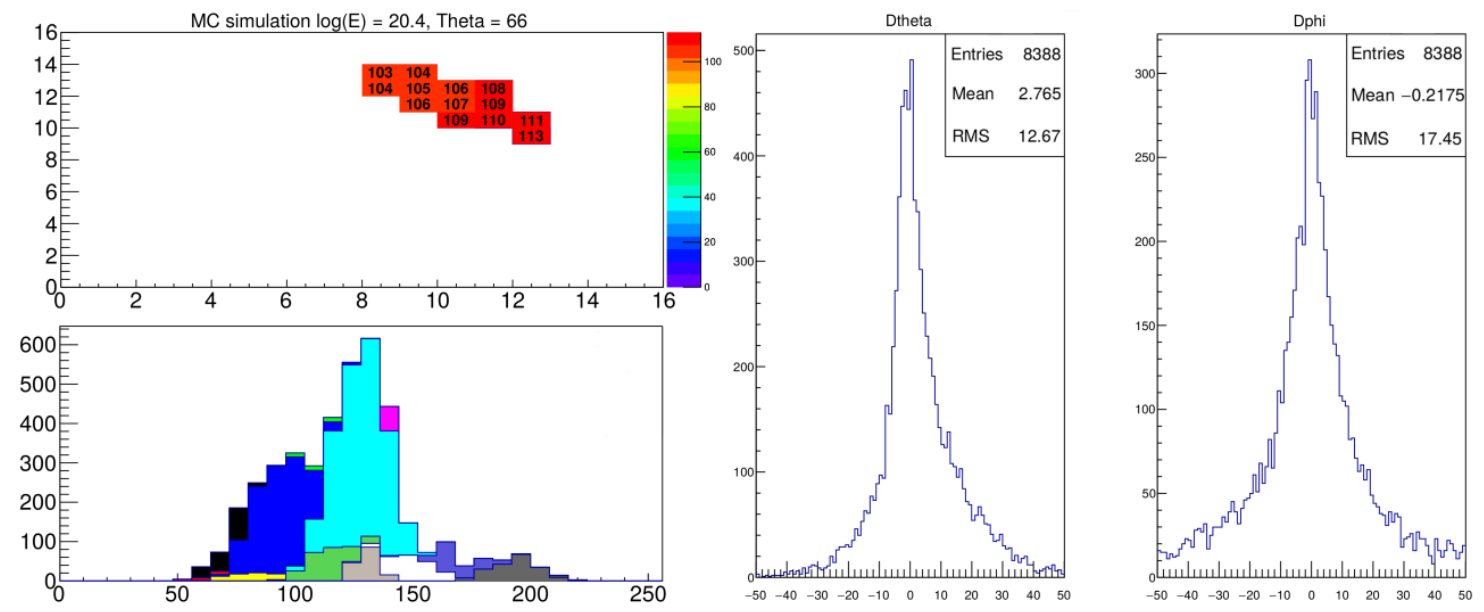

Figure 3 Left: The track of the simulated EAS on the PMT matrix (upper panel) and EAS cascade curve (lower panel). Right: Preliminary results of the difference measurement between simulated and reconstructed zenith $\theta$ and azimuthal $\varphi$ angles.

The Monte-Carlo simulated events in the energy range $\log _{10}(\mathrm{E})[\mathrm{eV}]=19.7-20.5$ and zenith angles $\theta=0^{\circ}-80^{\circ}$ were analyzed in the reconstruction program to find a procedure based on the formula (3) for a zenith angle calculation (see below). A difference between the arrival zenith $\theta$ and azimuthal $\varphi$ angles of simulated EAS as given by ESAF + TUSSIM programs and the reconstructed angles for these events are presented in Fig.3 (right). Only those events were selected for the evaluation of the $\theta$ and $\varphi$ accuracy reconstruction which satisfy a condition for EAS tracks to be inside of the field of view. Sometimes the difference is quite large. One of the possible reasons for such difference is due to increasing the PSF function of the TUS mirror at the edges of the PMT matrix.

A much more difficult problem is an energy evaluation, even for simulation of EAS where the PMT calibration constants are known by definition. It is obvious that proper EAS energy measurements are possible only for the events, images of which on the PMT matrix are aside of its edges and not-working channels to be sure that all EAS parts are measured. Currently, the 
Monte-Carlo simulation events are used to develop its energy restoration and this work is in progress.

\section{EAS event search from TUS data and analysis.}

A few tens of EAS candidates were found with a procedure described above in the available data set and analyzed according to the understanding of the EAS physics and the TUS detector simulation. Some details of the analysis criteria may be found in a recent paper [16]. Analysis of TUS data with the TUSReadData program shows that most of the events cannot be the EAS candidates. It looks like a short $\sim 150 \mu$ s flash of light. Sometimes such events look like tracks of the few pixels length. The time evolution analysis which is similar to plots in Fig. 4 gives the signal propagation with a superluminal speed. Fit of hit pixels in the $(x, y, t)$ space gives the zenith angles near zero. It means that we have a non-moving flash source of light. There were no Cherenkov flashes at the ends of cascade curves as it may be expected in some EAS events. Besides the time durations of the signals are longer than it should be for vertical EASs. The pseudo EAS events distribution on the PMT matrix is homogeneous that excludes the apparatus nature for these events.

The results of the research of the best and the most convincing EAS candidate are presented in Figs. 4 - 6 (it has TUS trigger number \#161003_054859-487). In Fig.4 the summary of the event is shown: the date and UTC time of the event measurement are the figure title, hit pixels together with dead ones in the upper panel and the amplitude variation of time for selected hit pixels in the lower panel. The markers and colors of the selected pixels on the upper panel indicate the arrival times for the maximum EAS signals in the time steps. The length of the EAS projection on the ground is $15-20 \mathrm{~km}$ and EAS duration is about $150 \times 0.8=$ $120 \mu \mathrm{s}$ as follows from the bottom plot. The different colors of this plot correspond to the different pixel contributions to the full EAS signal.
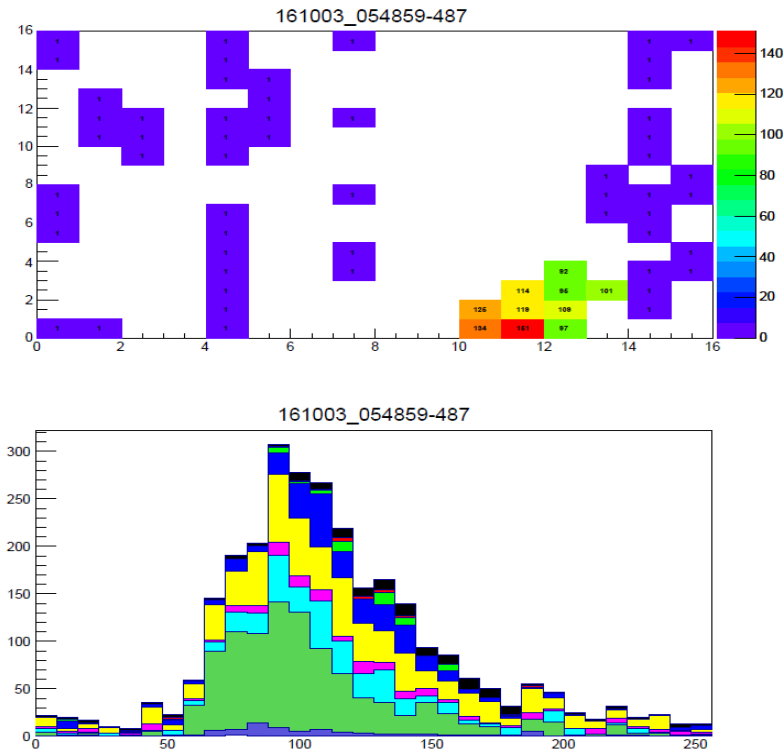

Figure 4. The EAS candidate with ten hit pixels. Upper plot - image of event with hit pixels and not-working (blue) ones. Bottom plot - the amplitude variation of time for selected hit pixels (stack histogram).

An important remark about EAS event in Fig.4 is the time sequence of hit pixels that corresponds to moving EAS signal towards the outside of PMT matrix which is a possible reason of the absence of the Cherenkov peak in the bottom plot. The time dependences of the EAS signals in the hit pixels are shown separately in Fig.5 with the Gauss function fit on top of it as described above. 

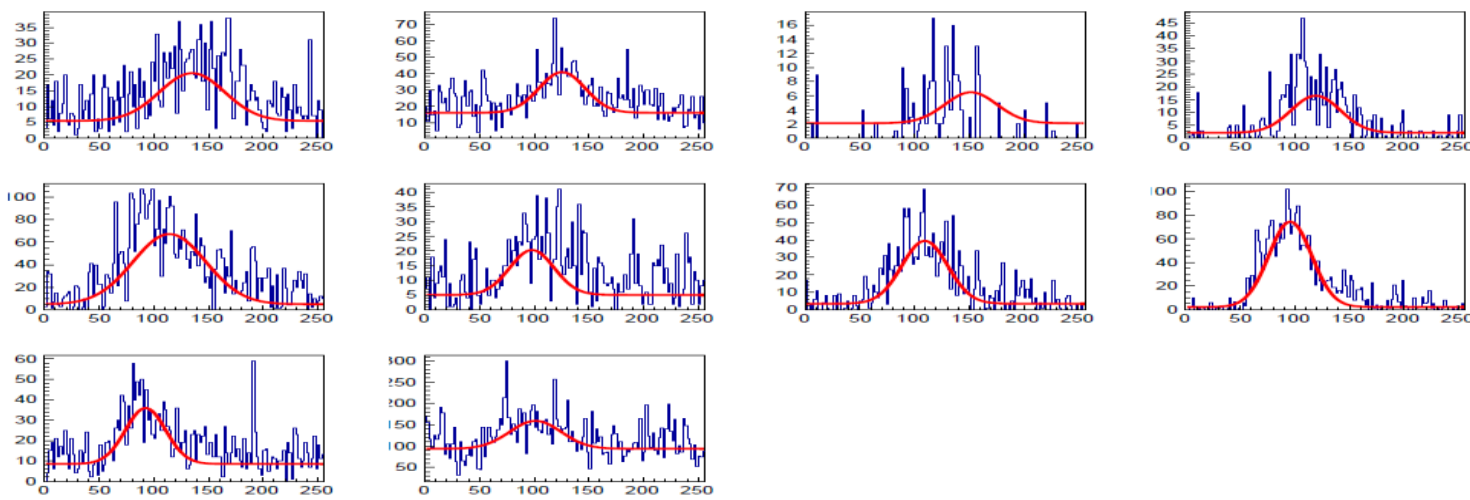

Figure 5. Time distributions of the EAS signals in the hit PMT pixels of the EAS candidate

event.

A more detailed statistical characteristics of the hit PMTs are presented in Table 1: "Module" and "PMT" are numbers of the hit module and PMT in the module, "Plot" is the plot number in Fig.5, Mean ${ }_{\mathrm{A}}$ and $\mathrm{RMS}_{\mathrm{A}}$ are the hit pixel Mean and RMS of the original amplitudes during the 256 time steps of the given event, "Bckg" is the $p_{0}$ - constant value of the fit, $A_{\max }$, $t_{\max }$ and $\sigma_{t}$ are the corresponding values of the Gaussian function fit (with goodness of fit as $\chi^{2}$ ).

Table 1. Statistical characteristics of the EAS event candidate \#161003_054859-487.

\begin{tabular}{|c|c|c|c|c|c|c|c|c|c|}
\hline Module & PMT & Plot & RMS $_{\mathrm{A}}$ & Mean $_{\mathrm{A}}$ & $\mathrm{Bckg}$ & $A_{\max }$ & $t_{\max }$ & $\sigma$ & $\chi^{2}$ \\
\hline 10 & 0 & 1 & 5.4 & 7.2 & 5.4 & 15.0 & 134.9 & 29.7 & 4.90 \\
10 & 1 & 2 & 7.3 & 12.5 & 16.0 & 24.8 & 125.5 & 20.9 & 4.07 \\
11 & 0 & 3 & 2.1 & 0.8 & 2.1 & 4.3 & 151.5 & 23.7 & 2.18 \\
11 & 1 & 4 & 5.9 & 3.5 & 2.1 & 14.4 & 119.0 & 22.5 & 4.66 \\
11 & 2 & 5 & 16.1 & 17.7 & 4.8 & 62.4 & 114.2 & 33.2 & 11.77 \\
12 & 0 & 6 & 5.5 & 6.5 & 5.0 & 15.3 & 97.9 & 20.2 & 5.58 \\
12 & 1 & 7 & 8.8 & 7.0 & 3.4 & 36.0 & 109.3 & 20.8 & 5.23 \\
12 & 2 & 8 & 12.8 & 9.7 & 2.2 & 72.4 & 95.7 & 19.5 & 5.22 \\
12 & 3 & 9 & 7.0 & 8.9 & 8.4 & 27.4 & 92.4 & 18.3 & 4.83 \\
13 & 2 & 10 & 20.8 & 56.1 & 93.7 & 65.4 & 101.1 & 23.5 & 12.78 \\
\hline
\end{tabular}

The next step of the EAS candidate analysis is a reconstruction of its arrival directions and the energy. First of all, the linear 3D-fit of hit pixels was done in the $(x, y, t)$ coordinate system. The azimuthal $\varphi$-angle is calculated by a simple formula

$$
\operatorname{tg}(\varphi)=\Delta y / \Delta x
$$

where $\Delta y, \Delta x$ are $y$-, $x$-projection of the EAS track in the TUS coordinate system as shown in the upper plot of Fig.4. The x-axis is along the PMT modules, $y$-axis is along the PMT pixels.

The zenith $\theta$-angle calculation is more complicated. If the EAS signal disk moves in the atmosphere with the velocity of light, the formula for a calculation of zenith angle of EAS for ideal detector is given by expression

$$
\operatorname{tg}(\theta / 2)=(\Delta r / \Delta t) / c
$$

where $\Delta r / \Delta t$ is EAS speed projection on the (x,y) surface of the Earth, $c$ is the velocity of light. The $\Delta r$ is the difference between the ends of the EAS track's projection on the $(x, y)$ plane as can be seen in the left plot of Fig.6 and $\Delta t$ value is the difference between the ends of the EAS track's projection on the $(x, t)$ or $(y, t)$ planes. In fact, formula (3) is valid approximately because of non-ideality of the TUS optics and has to be corrected. In such way the angles of the found EAS candidate are $\theta=\sim 30^{\circ}, \varphi=\sim 225^{\circ}$ in the TUS coordinate system. 

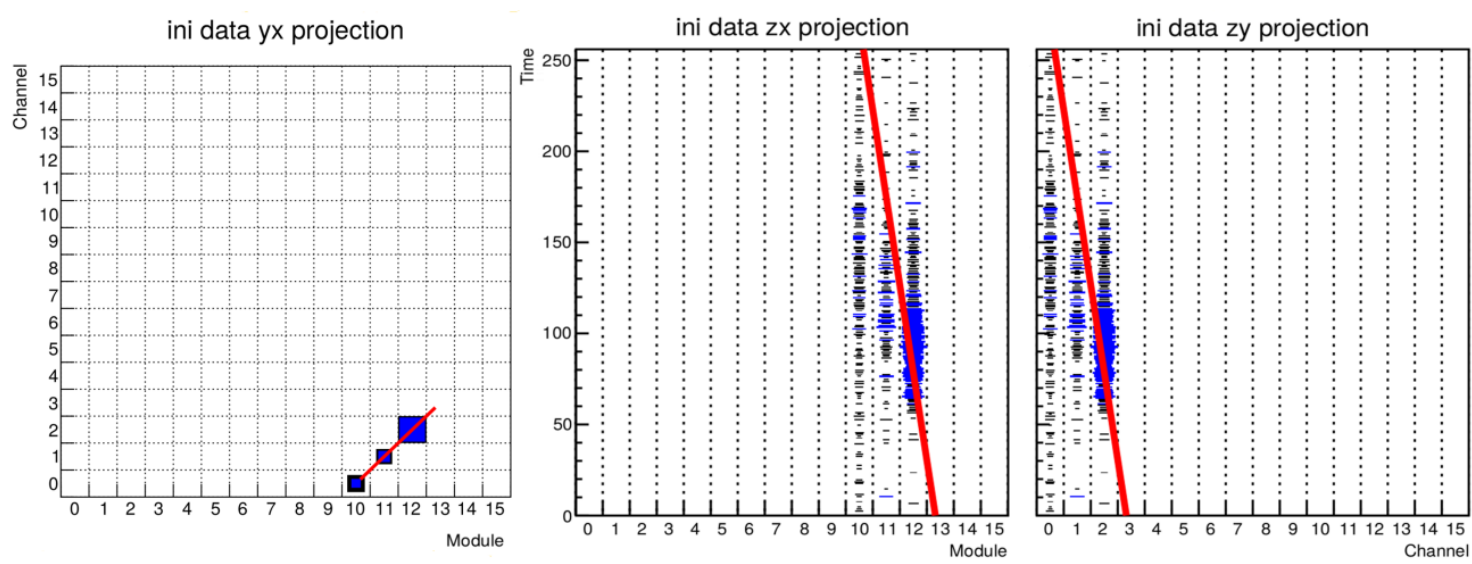

Figure 6. Linear 3D fit of the central EAS hit pixels for the EAS candidate event.

As was mentioned above, the difficult problem is the energy evaluation of the EAS candidate due to absence of reliably measured calibration constants for PMTs. According to Monte-Carlo simulation of the TUS detector [9] this energy has to be in the $100 \mathrm{EeV}$ region.

\section{Conclusion.}

The TUS detector is operating on board the "Lomonosov" satellite. During the search for an UHECR EAS a large number of events of various origins that take place in the atmosphere of the Earth were observed. These events may contain some genuine EAS events at energy $\sim 100$ EeV. A multi-level algorithm for the search of EAS-like events was developed and applied to the TUS data set analysis. As a result, at least one EAS candidate was selected and has been analyzed. A more detailed analysis of this and other EAS candidate events, which were found in the TUS detector data, is in progress.

\section{Acknowledgments}

The TUS experiment on board the "Lomonosov" satellite was realized within the Federal Space Program of Russia with funding by the Russian Space Agency. The data analysis is supported by RFBR grants No. 16-29-13065 and No. 15-02-05498.

\section{References}

[1] J. Linsley, Phys. Rev. Lett. 10, 146 (1963).

[2] A. Aab, P. Abreu, M. Aglietta, et al., Astrophys. J. 804, 15 (2015).

[3] P. Tinyakov, M. Fukushima, D. Ikeda, et al., Proc. of Science (ICRC2015), ID326 (2015).

[4] B. A. Khrenov et al., Nucl. Phys. B (Proc. Suppl.) 112 (2002) 115.

[5] B. A. Khrenov et al., Phys. Atom. Nucl. 67(11) (2004) 2058.

[6] Tsunesada Y 2013 Proc. 33rd Int. Cosmic Ray Conf. Rio de Janeiro (2013),

[7] Abbasi R U et al. Astropart. Phys. 64 (2015) 49 (Preprint arXiv:1408.1726).

[8] L.G. Tkatchev et al., 34th ICRC2015, PoS(ICRC2015)616.

[9] A. Grinyuk, V. Grebenyuk, B. Khrenov, et al.Astropart. Phys, 90:93-97, 2017.

[10] G. K. Garipov et al., Bull. Rus Acad. Sci Phys, 79(3):326328, 2015.

[11] The JEM-EUSO Collaboration, 40(1):1944, 2015. DOI: 10.1007/s10686-014-9418-x.

[12] Abrashkin et al., Advances in Space Research 37 (2006) 1867.

[13] P. A. Klimov et al., Lomonosov (UHECR/TLE Collaboration). ArXiv e-prints, 2017, 1706.04976.

[14] V.Grebenyuk at al., Physics of Particles and Nuclei Letters, 2016, Vol. 13, No. 5, pp. 579-582

[15] B.A. Khrenov at al., ArXiv e-prints, 2017:1704.07704v2

[16] S.V. Biktemerova et al., arXiv:1706.05369v1 [astro-ph.IM] 16 Jun 2017

[17] C. Berat, S. Bottai, D. De Marco, et al. Astroparticle Physics, 33:221\{247, May 2010, 0907.5275. 


\section{The Lomonosov-UHECR/TLE collaboration}

S.V. Biktemerova ${ }^{\text {, }}$, A.A. Botvinko ${ }^{\text {c }}$, A. I. Dmitrotsa ${ }^{\mathrm{g}}$, V.E. Eremeev ${ }^{\mathrm{a}}$, G.K. Garipov ${ }^{\mathrm{a}}$, V.M. Grebenyuk ${ }^{b ; d}$, A.A. Grinyuk ${ }^{b}$, S. Jeong ${ }^{\mathrm{e}}$, N.N. Kalmykova ${ }^{\mathrm{a}}$, M.A. Kaznacheeva ${ }^{\mathrm{a}}$, B.A. Khrenov ${ }^{\text {a }}$ M. Kime, P.A. Klimov ${ }^{\mathrm{a}}$, M.V. Lavrova ${ }^{\mathrm{b}}$, J. Lee ${ }^{\mathrm{f}}$, O. Martinez, M.I. Panasyuk ${ }^{\mathrm{a}}$, I.H. Park ${ }^{\mathrm{e}}$, V.L. Petrov ${ }^{\mathrm{a}}$, E. Ponce ${ }^{\mathrm{f}}$, A.E. Puchkov ${ }^{\mathrm{c}}$, H. Salazar ${ }^{\mathrm{f}}$, O.A. Saprykin ${ }^{c}$, A.N. Senkovsky ${ }^{c}$, S.A. Sharakin ${ }^{a}$, A.V. Shirokov ${ }^{\mathrm{a}}$, A.V. Tkachenko ${ }^{\mathrm{b}}$, L.G. Tkachev b;d, A. E. Volvach ${ }^{g}$, I.V. Yashin ${ }^{\mathrm{a}}$, M.Yu. Zotov ${ }^{\mathrm{a}}$

${ }^{a}$ M.V. Lomonosov Moscow State University, GSP-1, Leninskie Gory, Moscow, 119991, Russia bJoint Institute for Nuclear Research, Joliot-Curie, 6, Dubna, Moscow region, Russia, 141980 'Space Regatta Consortium, ul. Lenina, 4a, 141070 Korolev, Moscow region, Russia ${ }^{\mathrm{d} D u b n a}$ State University, University str., 19, Bld.1, Dubna, Moscow region, Russia eDepartment of Physics and ISTS, Sungkyunkwan University, Seobu-ro 2066, Suwon, 440-746 Korea ${ }^{f}$ Benemerita Universidad Autonoma de Puebla, 4 sur 104 Centro Historico C.P. 72000, Puebla, Mexico ${ }^{\mathrm{g}}$ Crimea Astrophysical Observatory, Simeiz, Russia 